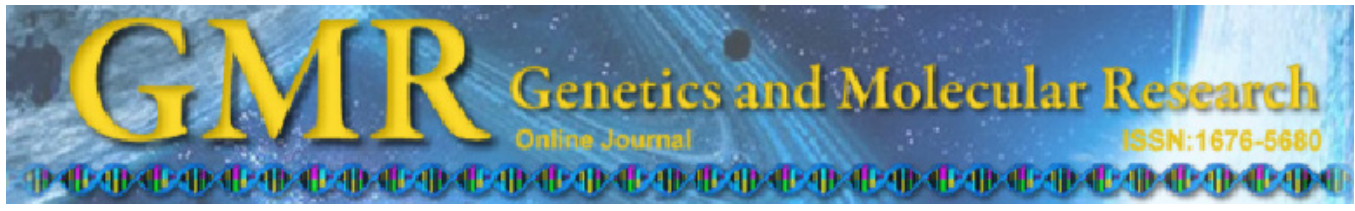

\title{
Age-dependent expression of the BPI gene in Sutai piglets
}

J. Zhu ${ }^{1}$, C. Zi ${ }^{1}$, Z.C. Wu ${ }^{1}$, L. Liu ${ }^{1}$, X.R. Zheng ${ }^{1}$, X.M. Su${ }^{1}$, G.Q. Zhu ${ }^{2}$, X.G. Huang ${ }^{3}$, S.L. Wu ${ }^{1}$ and W.B. Bao ${ }^{1}$

${ }^{1}$ College of Animal Science and Technology,

Key Laboratory for Animal Genetics, Breeding,

Reproduction and Molecular Design of Jiangsu Province,

Yangzhou University, Yangzhou, China

${ }^{2}$ College of Veterinary Medicine, Yangzhou University, Yangzhou, China

${ }^{3}$ Suzhou Taihu Pig Breeding Center, Jiangsu Province, Suzhou, China

Corresponding authors: W.B. Bao / S.L. Wu

E-mail: wenbinbao74@yahoo.com

Genet. Mol. Res. 12 (2): 2120-2126 (2013)

Received June 29, 2012

Accepted November 8, 2012

Published April 12, 2013

DOI http://dx.doi.org/10.4238/2012.April.12.1

\begin{abstract}
We compared and analyzed expression of the BPI gene of Sutai piglets ranging from newborn to post-weaning days 8 , 18,30 , and 35 by the real-time PCR method, in order to determine if it is involved in protection against disease caused by ETEC F18. There was a significant difference between 18- and 35-day expression in the jejunum. There were also significant differences between 35-day expression and expression at the other development stages in the duodenum. There were no significant differences in expression at 8,18 , and 30 days in the jejunum. We conclude that the porcine $B P I$ gene may be the direct factor that caused resistance to ETEC F18 in weaning piglets, and that the resistance to ETEC F18 in weaning piglets is related to upregulation of mRNA expression of the $B P I$ gene to a certain extent.
\end{abstract}

Key words: BPI gene; Gene expression; Pig; Real-time PCR 


\section{INTRODUCTION}

Bactericidal/permeability-increasing protein (BPI) is an endogenous cationic protein in humans and other mammals that is primarily found in the aniline blue particles of polymorphonuclear leukocytes. Among antibacterial peptides, BPI is notable for its high affinity (nM) for the lipid A region common to the LPS (lipopolysaccharide in Gram-negative bacteria (Levy, 2004). In addition to its capability to kill Gram-negative bacteria through neutralization of endotoxins or LPS, BPI has a series of biological functions such as opsonic function, promoting complement activation, opsonization for increased phagocytosis, inhibiting angiogenesis, inhibiting the release of inflammatory mediator, anti-fungal and protozoan, and it plays a critical role in the natural defenses of animals (Iovine et al., 1997; Elsbach, 1998). Christopher et al. (2004) have identified BPI as the candidate gene for the susceptibility of swine to Salmonella. Differential expression of BPI between enterotoxigenic Escherichia coli F18 (ETEC F18)-resistant and ETEC F18-sensitive piglets was analyzed in our preliminary study, and we determined that expression of $B P I$ is significantly high only in the duodenum and jejunum of pigs, suggesting that increased expression of $B P I$ in the intestines is connected to resistance to ETEC F18 (Ye et al., 2011).

Diarrhea and edema are two major infectious diseases that cause the death in postweaning piglets and lead to enormous economic losses in swine agriculture. Fimbrial adhesions produced by ETEC that bind to porcine mucosa include K88 (F4), K99 (F5), 987P (F6), F41 (F7), and F18 (Wilson and Francis, 1986; Imberechts et al., 1997). Of these, ETEC strains that produce $\mathrm{K} 88$ adhesions are the main cause of diarrhea in newborn piglets (Grange et al., 1998). Previous studies have confirmed that ETEC F18 is the main pathogen responsible for porcine post-weaning diarrhea and porcine edema disease (Nagy et al., 1990; Wittig et al., 1995). The analysis of expression at regular early time points is important, as newborn piglets are known to have diarrhea as a result of ETEC F4 infection during the first week of life and, at post-weaning, are vulnerable to diarrhea and edema caused by ETEC F18 infection (Bertschinger et al., 1990). The molecular epidemiology of porcine diarrhea has shown that ETEC F4 receptors are mainly expressed in the small intestine of newborn piglets, whereas ETEC F18 receptors are mainly expressed in piglets around the time of weaning, as the receptor plays a crucial role in mediating the binding of fimbriated bacteria to the intestinal epithelium at this developmental point.

We used a real-time polymerase chain reaction (PCR) method to compare and analyze expression of $B P I$ in piglets at various periods, aiming to determine whether $B P I$ expression has temporal and spatial specificity and elucidate the relationship between $B P I$ expression and sensitivity to various $E$. coli subtypes. This study provides a foundation for further research in the breeding of ETEC-resistant pigs.

\section{MATERIAL AND METHODS}

\section{Test materials}

The Sutai pig is a new hybrid of the Duroc and Taihu breeds. It produces high-quality lean meat. In 1999, this pig was approved by the National Committee of Livestock and Poultry Species as a new breed. Twenty-four Sutai pigs resistant to ETEC F18, raised under the same 
conditions, and healthy piglets aged $8,18,30$, and 35 days (6 individuals per age; weaning time, 30 days) were selected for tissue material sampling. The tissue samples of 11 organs, including the heart, liver, spleen, lung, kidney, stomach, muscle, thymus gland, lymph nodes, duodenum, and jejunum were collected. Samples were stored in liquid nitrogen on site and then transferred to a $-70^{\circ} \mathrm{C}$ freezer.

\section{Design and synthesis of real-time PCR primers}

Real-time PCR primers were selected according to published porcine $B P I$ and glyceraldehyde 3-phosphate dehydrogenase $(G A P D H)$ gene sequences. Using the Primer Express 2.0 software, we designed BPI primers based on the GenBank sequence NM_001159307. These primers were synthesized by Shanghai Invitrogen Biotechnology Co., Ltd. A 136-bp $B P I$ fragment was amplified with forward primer: 5'-ATATCGAATCTGCGCTCCGA-3' and reverse primer: 5'-TTGATGCCAACCATTCTGTCC-3'. Primers were designed across the exon to avoid genomic DNA contamination. GAPDH was used as an internal reference (forward primer: 5'-ACATCATCCCTGCTTCTACTGG-3'; reverse primer: 5'-CTCGGACGCCT GCTTCAC-3'. Its amplified fragment length was $187 \mathrm{bp}$.

\section{Total RNA extraction and real-time PCR}

RNA was extracted from the piglet tissues using a Trizol RNA extraction kit (Invitrogen, Gaithersburg, MD, USA) following manufacturer instructions. After electrophoresis with $1 \%$ agarose gel, RNA purity and concentration were determined using ultraviolet spectrophotometry, and the material was stored at $-70^{\circ} \mathrm{C}$. For complementary DNA synthesis, a $10-\mu \mathrm{L}$ reaction mixture containing $2 \mu \mathrm{L} 5 \mathrm{X}$ PrimerScript Buffer reaction mixture, $0.5 \mu \mathrm{L}$ PrimerScript RT Enzyme Mix I, $0.5 \mu \mathrm{L}$ Oligo dT, $0.5 \mu \mathrm{L}$ Random 6 mers, 500 ng total RNA, and RNasefree $\mathrm{H}_{2} \mathrm{O}$ up to $10 \mu \mathrm{L}$. Reaction conditions were set at $37^{\circ} \mathrm{C}$ for $15 \mathrm{~min}$ and $85^{\circ} \mathrm{C}$ for $5 \mathrm{~s}$, and the product was preserved at $4^{\circ} \mathrm{C}$.

\section{Fluorescent quantitative PCR system and reaction conditions}

The reaction system was $20 \mu \mathrm{L}: 1 \mu \mathrm{L}$ complementary DNA, $10 \mu \mathrm{M}$ up- and downstream of each primer, $0.4 \mu \mathrm{L} 50 X$ ROX Reference Dye II, $10 \mu \mathrm{L} 2$ X SYBR Green Realtime PCR Master Mix, and $7.8 \mu \mathrm{L}$ double-distilled $\mathrm{H}_{2} \mathrm{O}$. The PCR conditions were $95^{\circ} \mathrm{C}$ for $15 \mathrm{~s} ; 40$ cycles of $95^{\circ} \mathrm{C}$ for $15 \mathrm{~s}$ and $62^{\circ} \mathrm{C}$ for $34 \mathrm{~s}$; and preservation at $4^{\circ} \mathrm{C}$. The melting curve was analyzed after amplification. The unity of PCR amplification was determined using the melting curve $85^{\circ} \pm 0.8^{\circ} \mathrm{C}$ peak melting temperature. Each sample was tested 3 times using real-time PCR, and the average was calculated.

\section{Statistical analysis}

The $2^{-\Delta \Delta C t}$ method was used to process the real-time PCR results (Schmittgen et al., 2000). Statistical analyses were carried out using the SPSS 15.0 software (SPSS Inc., Chicago, IL, USA), and a $t$-test was performed to analyze the differentiation significance of messenger RNA expression among the various piglet development stages. 


\section{RESULTS}

\section{Analysis of RNA purity and integrity}

The electrophoresis of the total RNA extracted from the tissues exhibited 28S, 18S, and $5 \mathrm{~S}$ bands with no significant band DNA contamination or degradation, which indicated that the extracted total RNA had high purity. RNA purity was evaluated with ultraviolet spectrophotometry. The $\mathrm{A}_{260} / \mathrm{A}_{280}$ of the samples was 1.8-1.9, which indicated a high-quality RNA extraction that could be used for subsequent tests.

\section{Expression levels of $B P I$ in various tissues from 8-, 18-, 30-, and 35-day-old piglets}

A SYBR Green real-time fluorescence quantitative PCR method was used to detect changes in BPI expression in 11 types of tissue. The internal reference gene GAPDH was used to normalize the expression level, define the $B P I$ expression level of heart tissue as 1 , and calculate the relative quantitative levels of gene expression in other tissues. The results showed very low or no expression of $B P I$ in heart, liver, spleen, lung, kidney, muscle, thymus, and lymph node tissues but expression in stomach, duodenal, and jejunal tissues. The expression levels in duodenum and jejunum were significantly different from those in the other organs (Figure 1).

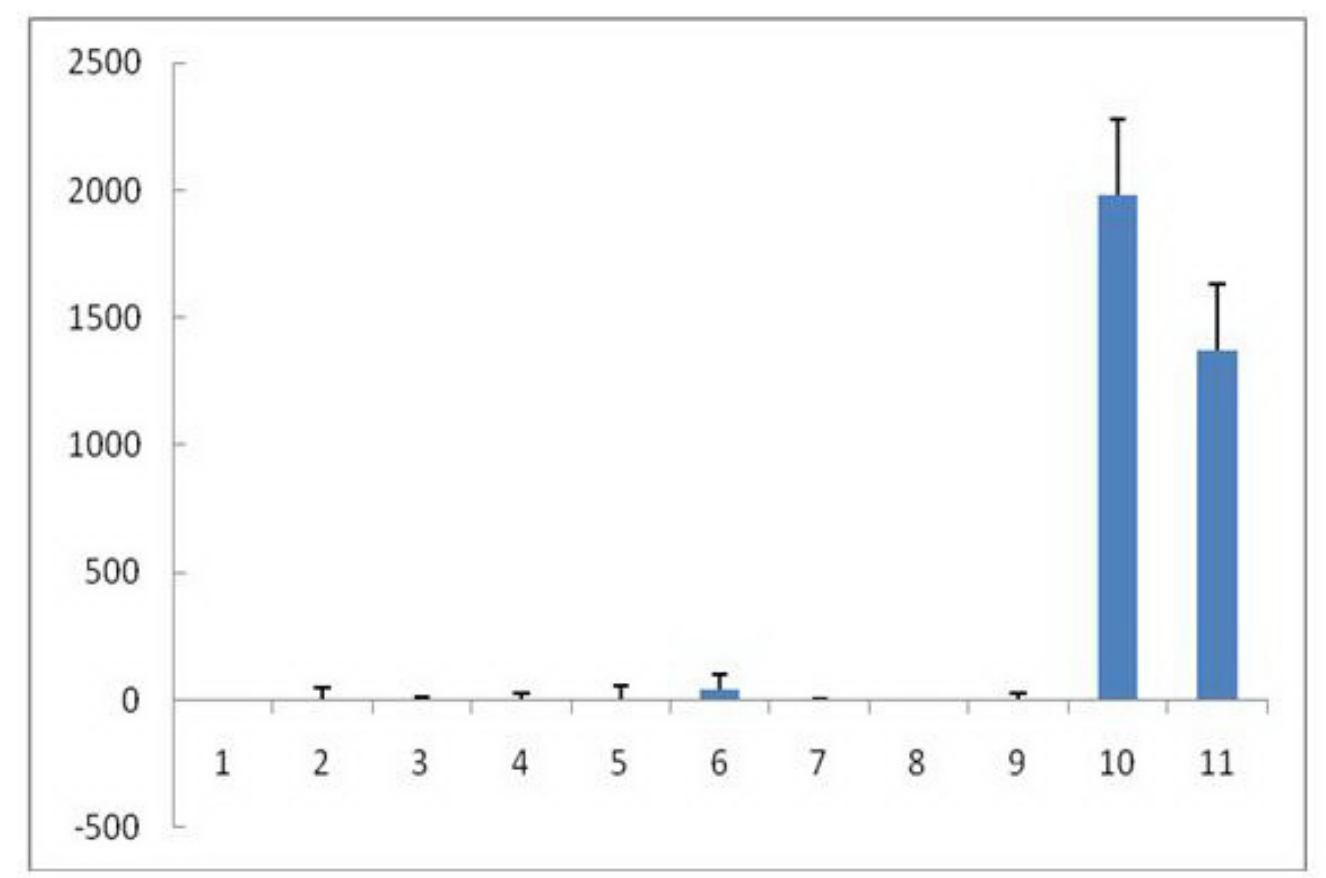

Figure 1. Expression of the BPI gene in various tissues. Columns 1-11 stand for heart, liver, spleen, lung, kidney, stomach, muscle, thymus, lymph, duodenum, and jejunum, respectively. 


\section{Amplification curve and melting curve of fluorescence quantitative PCR}

The PCR system automatically generated curves for cycle number and the detected changes in the amplification reaction of the fluorescence kinetics according to the changes of fluorescence. The BPI PCR amplification curve and melting curve are shown in Figure S1. The figure shows one specific peak in the BPI real-time PCR product, without primer dimer or the formation of non-specific products.

\section{Expression analysis of $B P I$ at various development stages in jejunum and duodenum}

We detected expression of $B P I$ in jejunum and duodenum at 4 development stages. Our study comprised 24 individuals, with 6 piglets each sampled at 8, 18, 30, and 35 days. The data indicated that average expression levels of BPI in jejunum and duodenum were present at the 4 development stages. Significant difference was found between days 18 and 35 in jejunum $(\mathrm{P}<0.05)$. In addition, significant difference occurred between day 35 and the other development stages in duodenum $(\mathrm{P}<0.05)$, but no difference was found among days 8,18 , and 30 in jejunum ( $>0.05$; Table 1, Figure $\mathrm{S} 2)$.

Table 1. Expression levels of the $B P I$ gene in jejunum and duodenum at different development stages.
\begin{tabular}{lcccc}
\hline Tissue & 8 days & 18 days & 30 days & 35 days \\
\hline Jejunum & $1.000 \pm 0.000^{\mathrm{ab}}$ & $0.454 \pm 0.133^{\mathrm{a}}$ & $1.796 \pm 1.365^{\mathrm{ab}}$ & $2.129 \pm 1.299^{\mathrm{b}}$ \\
Duodenum & $0.5198 \pm 0.342^{\mathrm{b}}$ & $0.195 \pm 0.067^{\mathrm{b}}$ & $0.432 \pm 0.288^{\mathrm{b}}$ & $3.001 \pm 1.781^{\mathrm{a}}$ \\
\hline
\end{tabular}

Numbers followed by different superscript letters within the same column mean significant differences at $\mathrm{P}<0.05$.

\section{DISCUSSION}

Neutrophils are a central component of the innate immune system of mammals. They are the first line of defense against foreign microorganisms, especially pathogens. BPI is a pluripotent protein in neutrophils and tissue that likely plays a pivotal role in host defense against Gram-negative bacteria and their endotoxins through antibiotic and endotoxin-neutralizing and disposing functions (Schultz et al., 2007). LPS is the main component in the outer membrane of Gram-negative bacteria. The crystal structure of BPI shows a boomerang-shaped, bi-partite molecule (Beamer et al., 1997) that includes a cationic, lysine-rich N-terminus containing the antibacterial and LPS (endotoxin)-neutralizing portions of the molecule (Ooi et al., 1991) and a C-terminus that contributes to the opsonic activity of BPI (Iovine et al., 1997). Because the N-terminal fragment of the BPI molecule is highly cationic, when BPI binds to lipid A of the LPS in the bacterial cell wall, the affinity and binding become very strong. Initially, the binding of BPI and the bacterial outer membrane activates a series of enzymatic systems, promoting the degradation of membrane phospholipids and peptidoglycan and changing the permeability and growth inhibition of the bacterial outer membrane. However, bacteria can also repair and grow. In the later stages of binding, the concentration of BPI increases as the length of time of activity increases, damaging the bacterial cell membrane. Structural and functional changes are induced, which lead to irreversible growth inhibition, and ultimately, lysis and death (Weiss et al., 1980; Mannion et al., 1990). 
Our study demonstrated that very low or no expression of $B P I$ occurs in heart, liver, spleen, lung, kidney, stomach, muscle, thymus, and lymph node tissues, but high levels of expression are found in duodenal and jejunal tissues that are significantly different from those of the other organs tested. Notably, BPI expression was barely observed in the spleen, lung, kidney, thymus, lymph nodes, and other immune organs. Therefore, BPI-induced ETEC F18 resistance is not achieved by regulating immune recognition. Combined with the biological activity of BPI, BPI is directly and closely related to resistance to intestinal ETEC F18 and other Gram-negative bacteria. When piglets are invaded by ETEC F18 strains, the bactericidal effects of BPI are activated. Because it has a cytotoxic effect on Gram-negative bacteria and neutralizes endotoxins, it kills ETEC F18 strains before they attach to the small intestine and release intestinal toxins, reducing the probability of diarrhea and edema in infected piglets. The tissues of both jejunum and duodenum were analyzed in our preliminary study, and the messenger RNA expression of $B P I$ in resistant individuals was significant higher than that in sensitive individuals $(\mathrm{P}<0.05)$. These results suggested that $B P I$ is likely related to the intestinal infection caused by ETEC F18. This increased expression of BPI in the intestine may be connected to resistance to ETEC F18 (Ye et al., 2011).

Coddens et al. (2007) reported that the expression level of the ETEC F18 strain receptor increases with piglet age during the first 3 weeks after birth and that expression is maintained in older pigs, which is consistent with the $B P I$ expression levels in our studies. Because the lowest level of duodenal $B P I$ expression was detected on days 8,18 , and 30 , these piglets are easily affected by ETEC F4 and similarly deleterious toxins, although owing to their weak specificity, they might not increase the expression level of $B P I$, suggesting that $B P I$ expression is stimulated by factors other than ETEC F4 itself. Given that ETEC F18 is responsible for inducing edema and diarrhea in post-weaning piglets, intestinal epithelial cells undergo constant stimulation by the toxins released by the pathogen, which may change the regulatory networks of $B P I$ and lead to higher expression. Therefore, it is reasonable to speculate that higher expression of $B P I$ on day 35 in duodenum is due to increased stimulation by ETEC F18.

This study demonstrates that porcine BPI plays a direct role in preventing ETEC F18 and $B P I$ expression is relevant to ETEC receptors, especially ETEC F18. Resistance to ETEC F18 in weaning piglets is related to the upregulation of $B P I$ expression in the intestinal tract.

\section{ACKNOWLEDGMENTS}

Research supported by the National Natural Science Funds (\#31172183), the Science and Technology Supporting Project (Agriculture) of Jiangsu Province (\#BE2010371, \#BE2012330) and Suzhou City (\#SN201133), and the Priority Academic Program Development of Jiangsu Higher Education Institutions

\section{Supplementary material}

\section{REFERENCES}

Beamer LJ, Carroll SF and Eisenberg D (1997). Crystal structure of human BPI and two bound phospholipids at 2.4 angstrom resolution. Science 276: 1861-1864.

Bertschinger HU, Bachmann M, Mettler C, Pospischil A, et al. (1990). Adhesive fimbriae produced in vivo by Escherichia coli O139:K12(B):H1 associated with enterotoxaemia in pigs. Vet. Microbiol. 25: 267-281. 
Christopher KT, Thomas JS, Shi XW and Martha AM (2004). Genetic Markers for Improved Disease Resistance in Animals (BPI). Patent Application: 20040234980, Kind Code: A1, Mckee Voorhees \& Sease PLC, United States.

Coddens A, Verdonck F, Tiels P, Rasschaert K, et al. (2007). The age-dependent expression of the F18 ${ }^{+}$E. coli receptor on porcine gut epithelial cells is positively correlated with the presence of histo-blood group antigens. Vet. Microbiol. 122: $332-341$.

Elsbach P (1998). The bactericidal/permeability-increasing protein (BPI) in antibacterial host defense. J. Leukoc. Biol. 64: 14-18.

Grange PA, Erickson AK, Anderson TJ and Francis DH (1998). Characterization of the carbohydrate moiety of intestinal mucin-type sialoglycoprotein receptors for the K88ac fimbrial adhesin of Escherichia coli. Infect. Immun. 66: 16131621.

Imberechts H, Bertschinger HU, Nagy B, Deprez P, et al. (1997). Fimbrial colonisation factors F18ab and F18ac of Escherichia coli isolated from pigs with postweaning diarrhea and edema disease. Adv. Exp. Med. Biol. 412: 175-183.

Iovine NM, Elsbach P and Weiss J (1997). An opsonic function of the neutrophil bactericidal/permeability-increasing protein depends on both its N- and C-terminal domains. Proc. Natl. Acad. Sci. U. S. A. 94: 10973-10978.

Levy O (2004). Antimicrobial proteins and peptides: anti-infective molecules of mammalian leukocytes. J. Leukoc. Biol. 76: 909-925.

Mannion BA, Weiss J and Elsbach P (1990). Separation of sublethal and lethal effects of polymorphonuclear leukocytes on Escherichia coli. J. Clin. Invest. 86: 631-641.

Nagy B, Casey TA and Moon HW (1990). Phenotype and genotype of Escherichia coli isolated from pigs with postweaning diarrhea in Hungary. J. Clin. Microbiol. 28: 651-653.

Ooi CE, Weiss J, Doerfler ME and Elsbach P (1991). Endotoxin-neutralizing properties of the $25 \mathrm{kD} \mathrm{N}$-terminal fragment and a newly isolated $30 \mathrm{kD} \mathrm{C}$-terminal fragment of the $55-60 \mathrm{kD}$ bactericidal/permeability-increasing protein of human neutrophils. J. Exp. Med. 174: 649-655.

Schmittgen TD, Zakrajsek BA, Mills AG, Gorn V, et al. (2000). Quantitative reverse transcription-polymerase chain reaction to study mRNA decay: comparison of endpoint and real-time methods. Anal. Biochem. 285: 194-204.

Schultz H, Hume J, Zhang dS, Gioannini TL, et al. (2007). A novel role for the bactericidal/permeability increasing protein in interactions of Gram-negative bacterial outer membrane blebs with dendritic cells. J. Immunol. 179: 2477-2484.

Weiss J, Elsbach P, Olsson I and Odeberg H (1978). Purification and characterization of a potent bactericidal and membrane active protein from the granules of human polymorphonuclear leukocytes. J. Biol. Chem. 253: 2664-2672.

Weiss J, Beckerdite-Quagliata S and Elsbach P (1980). Resistance of gram-negative bacteria to purified bactericidal leukocyte proteins: relation to binding and bacterial lipopolysaccharide structure. J. Clin. Invest. 65: 619-628.

Wilson RA and Francis DH (1986). Fimbriae and enterotoxins associated with Escherichia coli serogroups isolated from pigs with colibacillosis. Am. J. Vet. Res. 47: 213-217.

Wittig W, Klie H, Gallien P, Lehmann S, et al. (1995). Prevalence of the fimbrial antigens F18 and K88 and of enterotoxins and verotoxins among Escherichia coli isolated from weaned pigs. Zentralbl. Bakteriol. 283: 95-104.

Ye L, Zi C, Liu L, Zhu J, et al. (2011). Study on the relationship between the expression of BPI gene and Escherichia coli F18 infection in piglets. Yi Chuan 33: 1225-1230. 